\title{
Magnetic and superconducting phase diagrams in $\mathrm{ErNi}_{2} \mathrm{~B}_{2} \mathrm{C}$
}

\author{
J.A Galvis, M. Crespo, I. Guillamón, H. Suderow, S. Vieira, ${ }^{a}, 1$ M. García Hernández, b \\ S. Bud'ko and P.C. Canfield ${ }^{\mathrm{c}}$ \\ ${ }^{a}$ Laboratorio de Bajas Temperaturas, Departamento de Física de la Materia Condensada \\ Instituto de Ciencia de Materiales Nicolás Cabrera, Facultad de Ciencias \\ Universidad Autónoma de Madrid, 28049 Madrid, Spain \\ b Intituto de Ciencia de Materiales de Madrid, Consejo Superior de Investigaciones Científicas, Campus de Cantoblanco, \\ E-28049 Madrid, Spain. \\ ${ }^{\mathrm{c}}$ Ames Laboratory and Departament of Physics and Astronomy \\ Iowa State University, Ames, Iowa 50011, USA
}

\begin{abstract}
We present measurements of the superconducting upper critical field $\mathrm{H}_{c 2}(\mathrm{~T})$ and the magnetic phase diagram of the superconductor $\mathrm{ErNi}_{2} \mathrm{~B}_{2} \mathrm{C}$ made with a scanning tunneling microscope (STM). The magnetic field was applied in the basal plane of the tetragonal crystal structure. We have found large gapless regions in the superconducting phase diagram of $\mathrm{ErNi}_{2} \mathrm{~B}_{2} \mathrm{C}$, extending between different magnetic transitions. A close correlation between magnetic transitions and $\mathrm{H}_{c 2}(\mathrm{~T})$ is found, showing that superconductivity is strongly linked to magnetism.
\end{abstract}

Key words: A. Superconductors, A. Magnetic metals, D. Tunneling, D. Magnetic phase diagram

$\mathrm{ErNi}_{2} \mathrm{~B}_{2} \mathrm{C}$ is a prominent member of the series of the quaternary rare earth nickel borocarbides $\left(\mathrm{RNi}_{2} \mathrm{~B}_{2} \mathrm{C}\right.$ with $\mathrm{R}=\mathrm{Gd}-\mathrm{Lu}$ and $\left.\mathrm{Y}\right)$, because of the coexistence of superconductivity with different kinds of magnetic ordering in an accessible temperature and magnetic field range[1-3]. It becomes superconducting below about $11 \mathrm{~K}$, goes into an incommensurate antiferromagnetic (AF) phase below $\mathrm{T}_{N}=6.3 \mathrm{~K}$, and develops a net weak ferromagnetic component (WF) below $\mathrm{T}_{W F}=2.3 \mathrm{~K}$, where one of each twenty spins of the initially fully antiferromagnetic modulation are aligned parallel to each other [4-10]. Remarkably, superconductivity survives all magnetic transitions, contrary to what occurs in the Chevrel phase compounds $\left(\mathrm{RRh}_{4} \mathrm{~B}_{4}\right.$ with $\mathrm{R}$ being a rare earth), where a reentrance to the normal phase is observed when entering a ferromagnetic state[11-15]. One of the most salient features is a

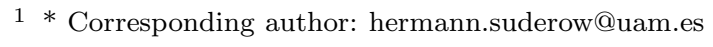

strong interrelationship between the magnetic and superconducting phase diagrams, first observed in the superconducting upper critical field[8]. The magnetic phase diagram has been studied using a variety of techniques. Neutron scattering and magnetization data have revealed the form of the spin alignment in a wide range of magnetic fields and temperatures [16,17]. A magnetoelastic tetragonal to orthorhombic distortion has been observed at $\mathrm{T}_{N}$ using synchroton X-ray scattering[18], and the corresponding anomalies in the thermal expansion have been followed in ref.[19]. Thermal expansion, magnetostriction, and specific heat measurements have revealed insight into the pressure dependence of the transitions[20]. Scanning Hall probe microscopy has revealed the presence of a small random magnetic field all over the surface of this compound [21].

The superconducting electronic density of states of $\mathrm{ErNi}_{2} \mathrm{~B}_{2} \mathrm{C}$, which is unaccessible from specific 
heat due to the overwhelmingly large magnetic contributions [20,22], has been studied using scanning tunneling microscopy and spectroscopy. Instead of a well developed BCS superconducting density of states a high amount of excitations appear at the Fermi level, with a $\mathrm{V}$-shaped density of states, which persists all the way up to $\mathrm{T}_{c}[23]$, pointing towards a highly anomalous superconducting density of states. $\mathrm{TmNi}_{2} \mathrm{~B}_{2} \mathrm{C}$ orders antiferromagnetically below 1.5 $\mathrm{K}$, with a far smaller local magnetic moment and has a well developed superconducting gap [24]. Pair breaking due to strong magnetic scattering, has been argued to be more important in $\mathrm{ErNi}_{2} \mathrm{~B}_{2} \mathrm{C}$ than in $\mathrm{TmNi}_{2} \mathrm{~B}_{2} \mathrm{C}$ due to the stronger exchange field in the former compound[25]. Recent thermal conductivity measurements in $\mathrm{HoNi}_{2} \mathrm{~B}_{2} \mathrm{C}$ have shown that enhanced magnetic pair breaking exists in a large part of the phase diagram, with superconducting features in the thermal conductivity appearing below the resistively measured upper critical field[26].

The objective of the present work is to measure $\mathrm{H}_{c 2}(\mathrm{~T})$ of $\mathrm{ErNi}_{2} \mathrm{~B}_{2} \mathrm{C}$ using tunneling spectroscopy, and search for possible relationships to the magnetic phase diagram. We have used similar experimental set-up and samples as in previous works [23,27,28], consisting of a STM in a dilution refrigerator. Here we use gold tips, and the sample was prepared by cutting a suitable needle out of single crystalline platelets of several $\mathrm{mm}$ thickness and one $\mathrm{cm}^{2}$ cross section oriented along the basal plane. The needles were broken in-situ, at low temperatures, providing for clean surfaces. The magnetic field was applied parallel to the tunneling direction, i.e. along the a axis. Topography and spectroscopic features have been discussed elsewhere[27,29]. Identification of magnetic transitions with STM using the length changes produced by the thermal expansion of the sample was discussed previously in Refs.[27]. We follow changes in the position of the tip over the surface of the sample of $\mathrm{ErNi}_{2} \mathrm{~B}_{2} \mathrm{C}$, when varying the temperature[30]. We observe strong changes close to magnetic transitions, related to the longitudinal thermal expansion along the a axis. In Fig.1 we show anomalies at the magnetic transitions, giving clear-cut peaks with a height comparable to results obtained with other methods[20,19]. The position of the peaks give the magnetic transition temperatures, which are comparable to previous work.

To measure the upper critical field, we follow the superconducting tunneling features as a function of temperature and magnetic field, in different positions on the surface[31]. The superconducting fea-
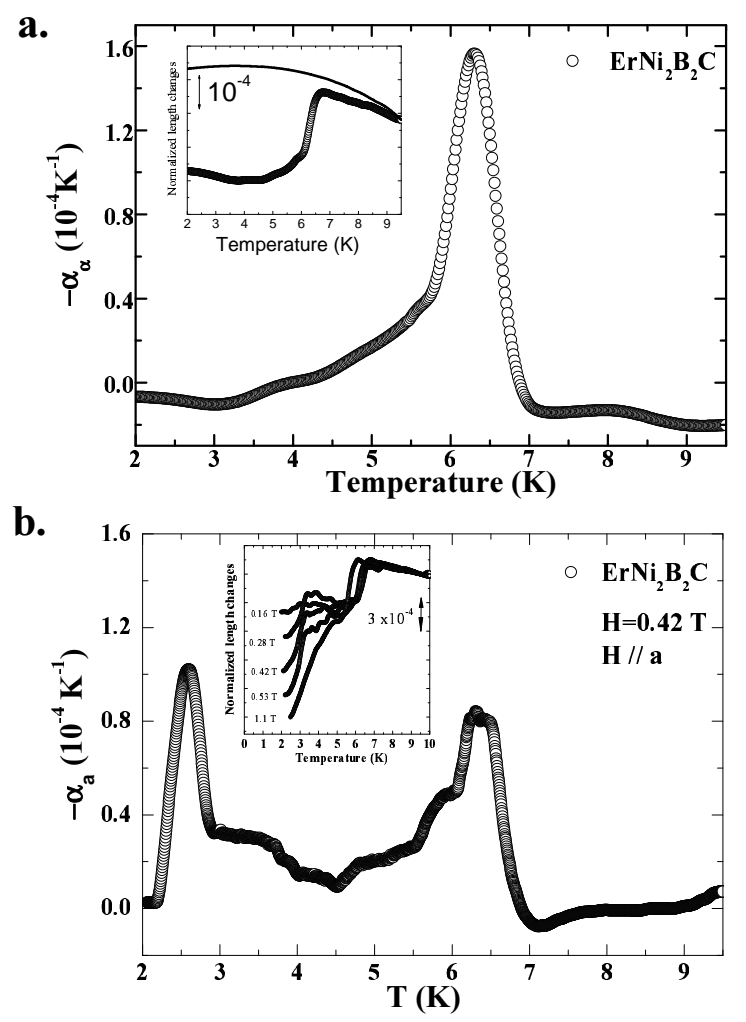

Fig. 1. The longitudinal thermal expansion of the sample perpendicular to the basal plane $\alpha_{a}$, as a function of temperature at zero field (a) and under magnetic fields (b). In the inset of (a) we show the changes in the $\mathrm{z}$ position of the tip, together with the result obtained in a similar sample of $\mathrm{TmNi}_{2} \mathrm{~B}_{2} \mathrm{C}$. In the inset of (b) we show a representative example of the $\mathrm{z}$ position as a function of temperature at (from top to bottom) $0.16 \mathrm{~T}, 0.28 \mathrm{~T}, 0.42 \mathrm{~T}, 0.53 \mathrm{~T}$ and $1.1 \mathrm{~T}$.

tures are weak in $\mathrm{ErNi}_{2} \mathrm{~B}_{2} \mathrm{C}$, so we did not resolve the vortex lattice[29]. We trace the temperature of field dependence of the zero bias conductance, normalized to its value at high bias. In the insets of Fig.2 we show some representative tunneling conductance curves in $\mathrm{ErNi}_{2} \mathrm{~B}_{2} \mathrm{C}$ and in the nonmagnetic parent compound $\mathrm{LuNi}_{2} \mathrm{~B}_{2} \mathrm{C}$. We define $\mathrm{H}_{c 2}(\mathrm{~T})$ as the point where the normalized zero bias conductance (Fig.2) reaches 0.99 when increasing temperature or magnetic field. At this point, the tunneling curves are essentially flat and voltage independent below some $10 \mathrm{mV}$, within the experimental uncertainty. In the Fig. 3 we show $\mathrm{H}_{c 2}(\mathrm{~T})$ thus obtained. Note that although the data taken in $\mathrm{LuNi}_{2} \mathrm{~B}_{2} \mathrm{C}$ closely follow previous results obtained by tracing the resistive transition (Fig.3), there is a large difference between $\mathrm{H}_{c 2}(\mathrm{~T})$ found in $\mathrm{ErNi}_{2} \mathrm{~B}_{2} \mathrm{C}$ through tunneling spectroscopy and 


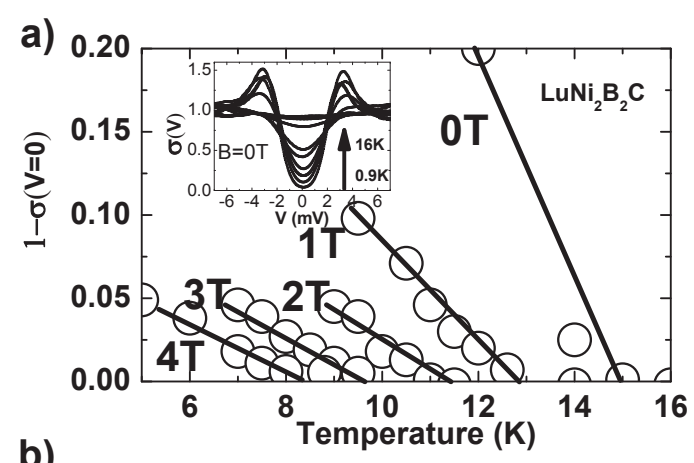

b)

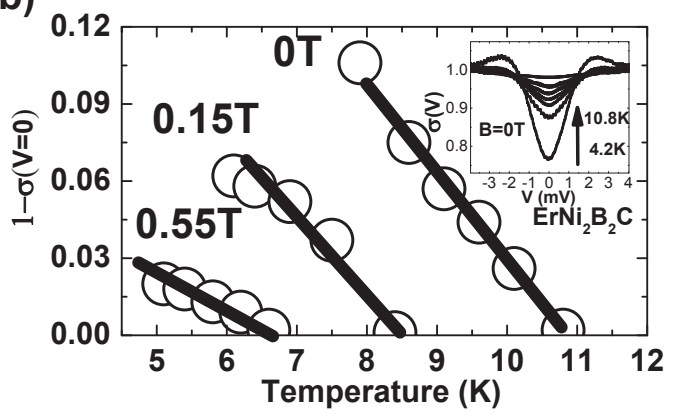

Fig. 2. We show $1-\sigma(\mathrm{V}=0)(\sigma(\mathrm{V}=0)$ is the normalized zero bias conductance) as a function of the temperature for $\mathrm{LuNi}_{2} \mathrm{~B}_{2} \mathrm{C}$ (a) and for $\mathrm{ErNi}_{2} \mathrm{~B}_{2} \mathrm{C}$ (b) at several magnetic fields. The insets show corresponding tunneling spectroscopy curves. The size of the points is about $1 \%$ of the high bias conductance and give experimental uncertainty. Lines are guides to the eye. The extrapolation of the lines to the $\mathrm{x}$-axis gives the critical temperature at the given magnetic fields. Similar curves are obtained when varying the magnetic field at a fixed temperature.

the result obtained by the resistive transition. This means that there is a large temperature and field range where $\mathrm{ErNi}_{2} \mathrm{~B}_{2} \mathrm{C}$ is essentially featureless in the tunneling conductance, still showing zero resistance. In $\mathrm{TmNi}_{2} \mathrm{~B}_{2} \mathrm{C}$, which has a transition to an antiferromagnetic state below $1.5 \mathrm{~K}$, and where the gap is fully open at low temperatures $[29,24,32$ 34 , we did not find differences between tunneling data and the resistive transition. This would imply that magnetism of $\mathrm{ErNi}_{2} \mathrm{~B}_{2} \mathrm{C}$ leads to a significant destruction of the superconducting gap in applied magnetic fields.

Note on the other hand that data in $\mathrm{ErNi}_{2} \mathrm{~B}_{2} \mathrm{C}$ have been taken down to very low temperatures, extending the range studied in previous work $[8,17,3]$. There is a significant positive curvature at high temperatures, characteristic of many nickel borocarbide superconductors. This strong positive curvature has been associated with the anisotropy of the Fermi surface [35-38]. There are features at $\mathrm{T}_{3}^{*} \approx 6 \mathrm{~K}$, $\mathrm{T}_{2}^{*} \approx 4 \mathrm{~K}$ and $\mathrm{T}_{1}^{*} \approx 2 \mathrm{~K}$. The features at $6 \mathrm{~K}$ and 2

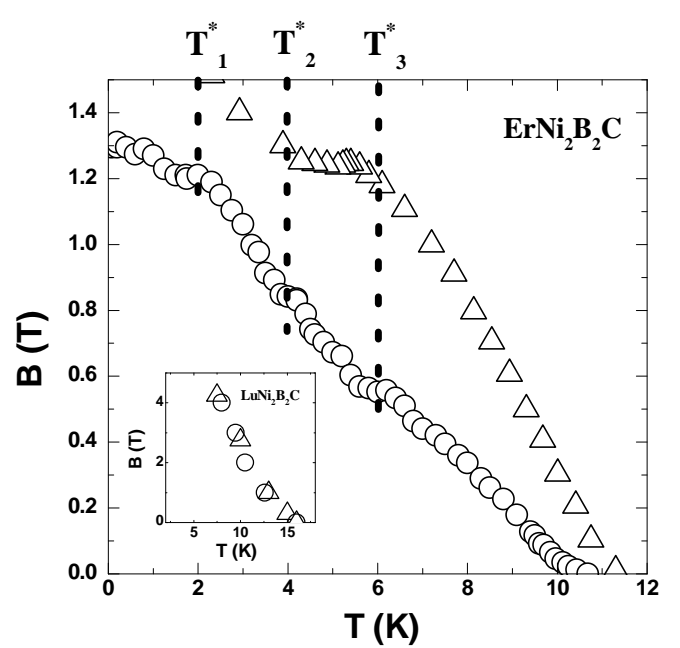

Fig. 3. The superconducting phase diagram of $\mathrm{ErNi}_{2} \mathrm{~B}_{2} \mathrm{C}$, down to $100 \mathrm{mK}$, with the field applied within the basal plane, obtained from local tunneling spectroscopy (open points) and from resistivity (triangles, taken from Ref.[16]). Note that Features in $\mathrm{H}_{c 2}(\mathrm{~T})$ are observed at approximately $\mathrm{T}_{1}^{*} \approx 2 K, \mathrm{~T}_{2}^{*} \approx 4 K$ and $\mathrm{T}_{3}^{*} \approx 6 K$ (dashed lines). Inset shows the same data (circles tunneling spectroscopy, triangles resistivity) in $\mathrm{LuNi}_{2} \mathrm{~B}_{2} \mathrm{C}$.

$\mathrm{K}$ are pronounced, and $\mathrm{H}_{c 2}(\mathrm{~T})$ becomes nearly flat at these points.

In Fig. 4 we compare the magnetic and superconducting phase diagrams in $\mathrm{ErNi}_{2} \mathrm{~B}_{2} \mathrm{C}$. As shown in Ref.[16], when a magnetic field is applied in plane between $\mathrm{T}_{W F}$ and $\mathrm{T}_{N}$, the initial incommensurate antiferromagnetic modulation $\left(\mathrm{Q}=0.55-0.56 \mathrm{a}^{*}\right)$, is modified into a field induced weak ferromagnetic state which locks to the lattice and becomes commensurate with a wavevector $\mathrm{Q}=(\mathrm{m} / \mathrm{n}) \mathrm{a}^{*}$, being $\mathrm{m}$ odd and $n$ even in most of the phases discussed in ref.[16]. When the magnetic field is increased, the wavelength of the commensurate structures is gradually reduced, and the number of spins aligned with the magnetic field increases. This produces new commensurate phases identified as well in neutron scattering measurements as in magnetization, which shows a steep increase at each transition $[8,17]$. The phases labeled in the Fig. 4 have been identified as the low field incommensurate AF phase $\mathrm{Q}_{1}=0.55$ $0.56 \mathrm{a}^{*}$, and the commensurate phases $\mathrm{Q}_{2}=0.57$ $\mathrm{a}^{*}, \mathrm{Q}_{3}=0.58 \mathrm{a}^{*}$ and $\mathrm{Q}_{4}=0.59 \mathrm{a}^{*}$. The final transition to the saturated paramagnetic state occurs at about $2 \mathrm{~T}$ along the a axis, for fields much above the loss of detectable superconducting gap via tun- 


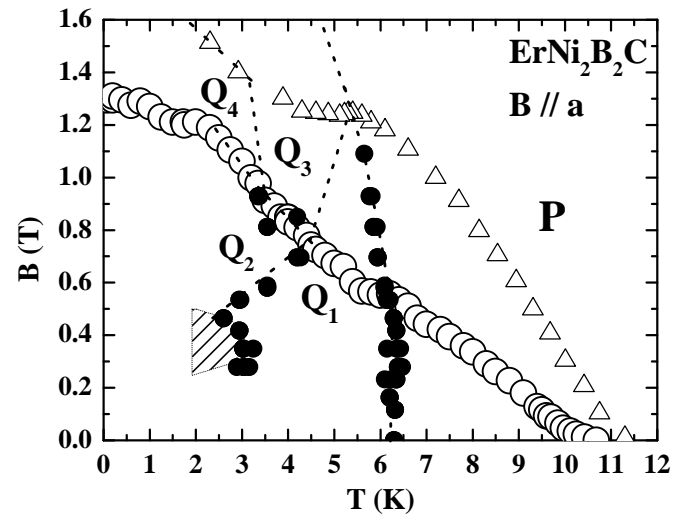

Fig. 4. The magnetic and superconducting phase diagrams of $\mathrm{ErNi}_{2} \mathrm{~B}_{2} \mathrm{C}$ with the field applied within the basal plane. Closed circles are points of the magnetic phase diagram obtained by thermal expansion (see also refs.[19,3]), and open circles the $\mathrm{H}_{c 2}(\mathrm{~T})$ data obtained by tunneling spectroscopy (same as Fig.2). Open triangles are $\mathrm{H}_{c 2}(\mathrm{~T})$ data obtained through resistivity, from ref.[16]. Dashed lines are the magnetic transitions viewed in neutron scattering experiments (from ref.[16]), and the different magnetic phases are labeled by $\mathrm{Q}_{1}-\mathrm{Q}_{4}[16]$. The magnetic states are labeled with $\mathrm{P}$ for paramagnetic state, and with the corresponding $\mathrm{Q}$ vector, determined in ref.[16], for the magnetic states.

neling spectroscopy. The features at $\mathrm{H}_{c 2}(\mathrm{~T})$ curve measured using tunneling spectroscopy at $\mathrm{T}_{2}^{*}$ (Fig. 3 ) actually coincides with a triple point (transition $\left.\mathrm{Q}_{1}-\mathrm{Q}_{2}-\mathrm{Q}_{3}\right)$ in the magnetic phase diagram. The rest of the $\mathrm{H}_{c 2}(\mathrm{~T})$ curve obtained using tunneling spectroscopy follows the magnetic transitions $\mathrm{Q}_{2}-$ $\mathrm{Q}_{3}$ and $\mathrm{Q}_{2}-\mathrm{Q}_{4}$ from $4.5 \mathrm{~K}$ to $2 \mathrm{~K}$. Below about 2 $\mathrm{K}$, another feature with a vanishing slope is observed in $\mathrm{H}_{c 2}(\mathrm{~T})$ curve obtained using tunneling spectroscopy. This possibly coincides with another magnetic transition, evolving from the low temperature weakly ferromagnetic state which exists at zero field. Note that the extended gapless region with zero resistance (Fig.4) is bound within the magnetic transitions $\mathrm{Q}_{2}-\mathrm{Q}_{4}$, showing that the density of states in this compound is significantly influenced by the underlying magnetic state. Thus, possible explanations for the gapless state can be enhanced magnetic scattering near the surface[25], or band-dependent strong pair breaking[26].

The strong correlation between magnetism and superconductivity in $\mathrm{ErNi}_{2} \mathrm{~B}_{2} \mathrm{C}$ leads to remarkable features in the superconducting phase diagram, which closely follows the changes in the local spin arrangement. The question of how these are linked together remains open for further study. Measurements of the vortex lattice, or of the tunneling spectroscopy using superconducting tips[39,40], should be useful, as well as other macroscopic measurements, such as thermal conductivity or London penetration depth.

\section{Acknowledgments.}

The Laboratorio de Bajas Temperaturas is associated to the ICMM of the CSIC. This work was supported by the U.S. Department of Energy, Office of Basic Energy Science, Division of Materials Sciences and Engineering. Part of this work was performed at the Ames Laboratory. Ames Laboratory is operated for the U.S. Department of Energy by Iowa State University under Contract No. DEAC02-07CH11358. This work was also supported by the spanish MICINN (grant FIS2011-23488, ACI2009-090 and Consolider Ingenio 2010 Nanociencia Molecular) and the Comunidad de Madrid through program Nanobiomagnet.

\section{References}

[1] P. C. Canfield, P. L. Gammel, D. J. Bishop, New magnetic superconductors: A toy box for solid-state physicists, Physics Today 51 (1998) 40.

[2] K. H. Müller, V. Narozhnyi, Interaction of superconductivity and magnetism in borocarbide superconductors, Reports Prog. Phys. 64 (2001) 943.

[3] S. Bud'ko, P. Canfield, Magnetism and superconductivity in the rare-earth nickel borocarbides, C.R. Physique 7 (2006) 56.

[4] R. Cava, H. Takagi, , B. Batlogg, H. Zandbergen, J. Krajewski, W. Peck, R. van Dover, R. Felder, T. Siegrist, K. Mizuhashi, J. Lee, H. Eisaki, S. Uchida, Superconductivity in the quaternary intermetallic compounds $\operatorname{lnni}_{2} b_{2} c$, Nature 367 (1994) 252.

[5] B. K. Cho, P. C. Canfield, L. L. Miller, D. C. Johnston, W. P. Beyermann, A. Yatskar, Magnetism and superconductivity in single-crystal erni $i_{2} b_{2} c$, Phys. Rev. B 52 (1995) 3684.

[6] J. Zarestky, C. Stassis, A. I. Goldman, P. C. Canfield, P. Dervenagas, B. K. Cho, D. C. Johnston, Magnetic structure of erni $i_{2} b_{2} c$., Physical Review B 51 (1995) R678.

[7] S. Sinha, J. Lynn, T. Grigereit, Z. Hossain, L. Gupta, R. Nagarajan, C. Godart, Neutron-diffraction study of antiferromagnetic order in the magnetic superconductor erni2b2c, Phys. Rev. B 51 (1995) 681.

[8] P. C. Canfield, S. L. Bud'ko, B. K. Cho, Possible coexistence of superconductivity and weak ferromagnetism in erni $i_{2} b_{2}$ c., Physica C 262 (1996) 249. 
[9] S. M. Choi, J. W. Lynn, D. Lopez, P. L. Gammel, P. C. Canfield, S. L. Bud'ko, Direct observation of spontaneous weak ferromagnetism in the superconductor erni $i_{2} b_{2} c$, Phys. Rev. Lett. 87 (2001) 107001.

[10] H. Kawano-Furukawa, H. Takeshita, M. Ochiai, T. Nagata, H. Yoshizawa, N. Furukawa, H. Takeya, K. Kadowaki, Weak ferromagnetic order in the superconducting erni $i_{2}^{11} b_{2} c$, Phys. Rev. B 65 (2002) 180508(R).

[11] E. O. Fisher, M. Maple, Superconductivity in ternary compounds, Vols I and II, Springer, New York.

[12] L. N. Bulaevski, A. I. Buzdin, M. L. Kulic, S. V. Panjukov, Coexistence of superconductivity and magnetism: Theoretical predictions and experimental results, Advances in Physics 34 (1985) $175-261$.

[13] M. Kulic, Conventional magnetic superconductors: coexistence of singlet superconductivity and magnetic order, C.R. Physique 7 (2006) 4.

[14] R. Prozorov, M. Vannette, S. Law, S. Bud'ko, P. Canfield, Physical Review B 77 (2008) 100503.

[15] V. Crespo, J. Rodrigo, H. Suderow, S. Vieira, D. Hinks, I. Schuller, Phys. Rev. Lett. 102 (2009) 237002.

[16] A. Jensen, K. N. Toft, A. B. Abrahamsen, D. F. McMorrow, M. R. Eskildsen, N. H. Andersen, J. Jensen, P. Hedegard, J. Klenke, S. Danilkin, K. Probes, V. Sikolenko, P. Smeibidl, S. L. Bud'ko, P. C. Canfield, Field-induced magnetic phases in the normal and superconducting states of $\mathrm{erni}_{2} \mathrm{~b}_{2} \mathrm{c}$, Phys. Rev. B 69 (2004) 104527.

[17] S. Bud'ko, P. Canfield, Rotational tuning of $H_{c 2}$ anomalies in erni $i_{2} c$ : Angular-dependent superzone gap formation and its effect on the superconducting ground state, Phys. Rev. B. 61 (2000) R14932.

[18] C. Detlefs, A. H. M. Z. Islam, T. Gu, A. I. Goldman, C. Stassis, P. C. Canfield, Magnetoelastic tetragonal-toorthorhombic distortion in erni $b_{2} b_{2}$ c, Phys. Rev. B. 56 (1997) 7843.

[19] M. Doerr, M. Rotter, M. Massalami, S. Sinning, H. Takeya, Magnetoelastic effects in erni $i_{2} b_{2} c$ single crystal: probing the h-t phase diagram, J. Phys.: Condens. Matter 14 (2002) 5609.

[20] S. Bud'ko, G. Schmiedeshoff, P. Canfield, Anisotropic thermal expansion and uniaxial pressure dependence of superconducting and magnetic transitions in $\operatorname{erni}_{2} \mathrm{~b}_{2} \mathrm{c}$, Solid State Comm. 140 (2006) 281.

[21] H. Bluhm, S. Sebastian, J. Guikema, I. Fisher, K. Moler, Scanning hall probe imaging of erni2b2c, Physical Review B 73 (2006) 014514.

[22] M. E. Massalami, R. E. Rapp, F. A. B. Chaves, H. Takeya, C. M. Chaves, Magnon specific heat of singlecrystal borocarbides $r n i_{2} b_{2} c(\mathrm{r}=\mathrm{tm}$, er, ho, dy, tb, gd), Phys. Rev. B 67 (2003) 224407.

[23] M. Crespo, H. Suderow, S. Bud'ko, P. C. Canfield, S. Vieira, Local superconducting density of states of $\mathrm{ErNi}_{2} \mathrm{~B}_{2} \mathrm{C}$, Phys. Rev. Lett. 96 (2006) 027003.

[24] H. Suderow, P. Martínez-Samper, S. Vieira, N. Luchier, J. P. Brison, P. C. Canfield, Tunneling spectroscopy in the magnetic superconductor $\mathrm{TmNi}_{2} \mathrm{~B}_{2} \mathrm{C}$, Phys. Rev. B 64 (2001) 020503(R).

[25] D. Gusakova, A. Golubov, M. Kupriyanov, A. Buzdin, Density of states in sf bilayers with arbitrary strength of magnetic scattering, Pis'ma V ZhETF 83 (2006) 385.
[26] M. Schneider, G. Fuchs, K.-H. Muller, K. Nenkov, G. Behr, D. Souptel, S. Drechsler, Physical Review B 80 (2009) 224522.

[27] M. Crespo, H. Suderow, S. Vieira, S. Bud'ko, P. C. Canfield, Thermal expansion measured by stm in the magnetic superconductor erni $_{2} b_{2}$ c, Physica B 378 (2006) 471.

[28] H. Suderow, I. Guillamon, S. Vieira, Rev. of Sci. Inst. 82 (2011) 033711.

[29] I. Guillamon, M. Crespo, H. Suderow, S. Vieira, J. Brison, S. Bud'ko, P. Canfield, Physica C 470 (2010) 771-775.

[30] J.-H. Park, D. Graf, T. Murphy, G. Schmiedeshoff, S. Tozer, Rev. Sci. Inst. 80 (2009) 116101.

[31] I. Guillamon, H. Suderow, A. Fernandez-Pacheco, J. Sese, R. Cordoba, J. D. Teresa, M. Ibarra, S. Vieira, Nature Physics 5 (2009) 651.

[32] M. R. Eskildsen, K. Harada, P. L. Gammel, A. B. Abrahamsen, N. H. Andersen, G. Ernst, A. P. Ramirez, D. J. Bishop, K. Mortensen, D. G. Naugle, K. D. D. Rathnayaka, P. Canfield, Intertwined symmetry of the magnetic modulation and the flux-line lattice in the superconducting state of $\mathrm{TmNi}_{2} \mathrm{~B}_{2} \mathrm{C}$, Nature 393 (1998) $242-245$.

[33] K. Noorgaard, M. R. Eskildsen, N. H. Andersen, Interdependence of magnetism and superconductivity in the borocarbide $\mathrm{TmNi}_{2} \mathrm{~B}_{2} \mathrm{C}$, Phys. Rev. Lett. 84 (2000) 4982 .

[34] K. Noorgaard, A. B. Abrahamsen, M. R. Eskildsen, K. Lefmann, N. H. Andersen, P. Vorderwisch, P. Smeibidl, M. Meissner, P. C. Canfield, Neutron diffraction study of anomalous high-field magnetic phases in $\mathrm{TmNi}_{2} \mathrm{~B}_{2} \mathrm{C}$, Phys. Rev. B. 69 (2004) 214507.

[35] S. V. Shulga, S.-L. Drechsler, G. Fuchs, K. H. Müller, K. Winzer, M. Heinecke, K. Krug, Upper critical field peculiarities of superconducting $y n i_{2} b_{2} c$ and $\operatorname{luni}_{2} b_{2} c$, Phys. Rev. Lett. 80 (1998) 1730.

[36] J. Brison, N. A. Sulpice, H. Suderow, P. Martínez-Samper, S. Vieira, A. Buzdin, P. Canfield, Anisotropic superconductivity in borocarbide superconductors and spin disorder, Journal of Magnetism and Magnetic Materials 272 (2004) 158.

[37] H. Suderow, V. G. Tissen, J. P. Brison, J. L. Martinez, S. Vieira, P. Lejay, S. Lee, S. Tajima, Pressure dependence of the upper critical field of $\mathrm{mgb}_{2}$ and of yni $_{2}$ b $_{2}$ c, Phys. Rev. B 70 (2004) 134518.

[38] H. Suderow, V. Tissen, J. Brison, J. Martinez, S. Vieira, Pressure induced effects on the Fermi surface of superconducting $2 \mathrm{H}-\mathrm{NbSe}_{2}$, Phys. Rev. Lett. 95 (2005) 117006.

[39] J. G. Rodrigo, H. Suderow, S. Vieira, E. Bascones, F. Guinea, Superconducting nanostructures fabricated with the scanning tunnelling microscope, J. Phys.: Condens. Matter 16 (2004) 1151.

[40] H. Suderow, V. Crespo, I. Guillamon, S. Vieira, F. Servant, P. Lejay, J. Brison, J. Flouquet, A nodeless superconducting gap in $\mathrm{Sr}_{2} \mathrm{RuO}_{4}$ from tunneling spectroscopy, New Journal of Physics 11 (2009) 093004. 